\title{
A Hybrid of Industrial Maintenance Decision Making Grids
}

\author{
Akilu Yunusa-Kaltungo ${ }^{a *}$, Ashraf Labib $^{b}$ \\ anniversity of Manchester, UK (corresponding author) \\ ${ }^{\mathrm{b}}$ University of Portsmouth, UK \\ E-mail addresses: akilu.kaltungo@ manchester.ac.uk (A. Yunusa-Kaltungo) \\ ashraf.labib@port.ac.uk (A. Labib)
}

Corresponding author: Akilu Yunusa-kaltungo

\begin{abstract}
It is fair to assume that the main challenge in maintenance decision making is the existence of a gap between theory and sustainable practice which is attributable to complexity, too much emphasis on development of new models that only serve to criticise earlier ones, underrepresentation of case studybased researches and lack of adequate incorporation of industry-based knowledge into most theoretical studies. In this paper, we revisited the application of the decision making grid (DMG) for maintenance optimisation but the main novelty here is to harmonise the strengths of the two most popular DMG approaches as opposed to the previous trends of advocating one over the other. Additionally, the current initiative limits assumptions associated with the process, since both DMG approaches depend on the main objective and nature of data involved. The data required for implementation are frequency of breakdown events and downtime for each event, which is readily available in all computerized maintenance management systems. This implies that we rely on just having access to some sort of a counter of faults and a timer for each event.
\end{abstract}

Keywords Manufacturing systems, industrial maintenance, decision analysis, decision making grid, maintenance strategies, case study 


\section{Introduction}

The industrial revolution significantly enhanced the abilities of manufacturing industries to improve the quality of human life through more precise and faster approaches that aided mass production. Traditional mass production principles were mainly based on the application of narrowly skilled experts, several unskilled workers, large inter-stage storage facilities and complex-single-purpose machines for generating huge volumes of standardized products (Melton 2005, Womack et al. 1990, Takata et al. 2004, Hines et al. 2004, Cowton and Vail 1994, Krafcik 1998). While this strategy immensely enhanced the abilities of manufacturing organisations to meet the sharp rise in the demands for products and services in the immediate post-war era, decision making was not a challenge owing to the abundance of resources and the readiness of consumers to always embrace what product/service providers threw at them. However, the shift in paradigm from a manufacturer-driven to a consumerdriven system soon led to the emergence of concepts such as lean or just-in-time (JIT) manufacturing (Kubiak and Yavuz 2008, Holweg 2007, Silver 2004). However, irrespective of whether manufacturing organisations adopt the traditional mass production or lean manufacturing approach for their processes, a common fundamental question that continuously emerges is "how do we guarantee that our plant assets are capable of effectively performing the functions for which they were acquired?"

A natural answer to this question has always been maintenance which can be described as the effective harmonisation of all managerial, technical and administrative processes that ensure that an industrial plant asset remains in or is quickly restored to a state of satisfactory operational performance (Yunusakaltungo et al. 2017, Yunusa-kaltungo and Sinha 2014). This definition is in close agreement with those offered by regulatory institutions including the British Standard Institute (BS 3811 1984) and European Standards on maintenance (EN 13306:2001, Marquez and Gupta 2006). Using an extract of the root cause failure analysis conducted on a major production incident that occurred on the most critical production unit within the manufacturing plant from which the research data used in the later parts of this study were obtained, the generic maintenance causal loop shown in Figure 1 reiterates how wrong/inadequate maintenance decisions led to asset malfunction which had immediate effects on several other vital plant parameters including plant throughput, product quality, equipment and personnel safety, finance, etc. The implications shown in Figure 1 are infinitesimal when compared to indirect long-term implications on customer satisfaction and reputation of the organisation.

Figure 1: A generic maintenance management causal loop

Despite the glaring alignment between business performance and the cost-effectiveness of maintenance management, the real-life perception of maintenance by organisations varies significantly between the spheres of useful-business-contributor and a necessary evil. However, the good news is that a common 
ground still exists whereby all organisations aiming to achieve sustained process excellence must constantly sort for mechanisms through which maintenance decisions can be optimised especially when it could account for as much as 15-40\% of production costs (Bevilacqua and Braglia 2000, Savsar 2006) depending on the particularly industry. An investigative study performed by Wireman (1990) and later supported by Chan (2005) on total productive maintenance further revealed that the total maintenance costs of just a few selected group of organisations exceeded 600 billion US dollars in a single year. The advocacy of the relevance of the maintenance function within organisations is nothing new, but in comparison to other business functions such as production, a lot more still has to be done (Al-Najjar 2007).

In the past, maintenance strategies were mainly breakdown maintenance (BM) and planned preventive maintenance (PPM) due to the high level of equipment redundancy and abundant materials that shrouded the earlier mass production eras. As the mantle of authority in modern day business environments is swiftly skewing towards the consumers, product makers are now required to meet highquality and significantly diverse consumer needs at lower costs. This is perhaps the reason for recent and sustained soar in the popularity of condition-based maintenance (CBM) strategy especially vibration monitoring (Al-Najjar 2007, Chilcott and Christer 1991, Wang et al. 2007, Jafari and Makis 2015, Yunusa-kaltungo and Sinha 2014), lube oil/wear debris analysis (Wang et al. 2012) and infrared thermography. While the achievements and progress of maintenance strategies such as CBM (YunusaKaltungo and Sinha, 2014; Guillen et al., 2016) are undeniable, typical manufacturing industries are often equipped with various assets which have different reliability and safety requirements. Hence the indiscriminate application of maintenance strategies (e.g. application of CBM to all plant assets or application of BM, PPM and CBM to a single plant asset) would be an utter waste of valuable resources and reoccurring failures (Yunusa-Kaltungo and Sinha, 2016; Seecharan et al. 2018, Moya 2004; Yunusa-Kaltungo and Sinha, 2012). Therefore, continuous enhancement of smart and easy-toimplement maintenance decision analysis systems such as the decision making grid (DMG) should form the nucleus of any maintenance management system that aims to contribute to overall business excellence. Despite the existing knowledge of DMG within the literature (Aslam-Zainudeen and Labib, 2011; Shahin et al., 2018; Fernandez et al., 2003; Seecharan et al., 2018; Tahir et al., 2009; Shahin et al., 2019; Herrera Galan, 2019), all previous studies on the tool have treated the two main approaches to boundaries determination as well as asset allocation to different maintenance strategies as discrete entities which may mislead users. This is primarily owing to the fact that the former approach (i.e. DMG1) is based on the principle of fixed and equal boundaries for both failure frequencies and downtime duration, which are always predefined by the user. The latter approach (i.e. DMG2) on the other hand adopts a tri-quadrant clustering method whereby criteria boundaries are governed by the sensitivity of the data. In the current paper, the authors recognise the validity of both lines of thoughts by harmonising DMG1 and DMG2. This is based on the premise that DMG1 could be used to ensure 
reasonable distribution of maintenance workload (or workload smoothening), which is always crucial to planning and resource allocation. Considering that large variations sometimes exist with regards to each of the criteria for different machines, DMG2 would suit such a scenario better as this will make the analysed classes of machines more comparable. Therefore, DMG1 is more ideal for workload smoothening while DMG2 is better suited for input data smoothening.

In addition to the novel combination of the most popular applications of DMG approaches presented, the case study based approach makes it draw inspiration from both historical trends of knowledge related to maintenance decision making theories within existing literature as well as the need to incorporate well-established/perfected skills such as Pareto histograms within the industry. It is envisaged that such combinations could enhance the possibility of academic-industry knowledge transfer in the near future without the need for drastic skill level upgrade (SLU), which has always proven to be a source of resistance to the deployment of novelty in practice. Hence, the remainder of the paper is structured thus. Studies related to maintenance decision analysis are reviewed in Section 2 so as to further buttress the point of directional skewness towards new techniques development and less towards harnessing existing industrial skills. Section 3 provides a brief overview of maintenance strategies as applied in decision making grid (DMG) so that the current article can be used as a standalone rather requiring constant reference to previously published DMG-related articles. Section 4 describes a framework for integrating existing DMG approaches for optimised maintenance strategies allocation. Section 5 provides details of the case studies as well as practical implementation of the proposed hybrid model. The findings from the paper are then concluded in Section 6.

\section{Historical trends of knowledge related to maintenance decision making theories}

Over the years, maintenance management researchers within the academia and industry have immensely contributed to the existing body of literature. A global keyword search ran by the authors of the current paper on industrial maintenance decision-making approaches for manufacturing systems (Goh et al., 2019; Roda et al., 2019; Ayu et al., 2019; Zhu et al., 2019; Cai et al., 2020; Nachimuthu et al., 2019; Lin et al., 2019; Dong et al., 2019; Li et al., 2019) reflects a very significant upsurge in the number of contributions directed towards this area which is anticipated to continue with the recent advances in computational technology as well as the increased realisation that real-life decision problems are seldom mono-criterion (Botti and Peypoch, 2013; Corrente et al., 2013; Wang and Triantaphyllou, 2008; Papadopoulos and Karagiannidis, 2008; Aguezzoul, 2014; Hartami-Marbini, 2011; Bumblauskas et al., 2017; Vujanovic et al., 2012; Yurin, 2012; Cassady et al., 2001; Carnero and Noves, 2006). Figure 2 details the search process adopted for identifying relevant studies. We searched for relevant articles without time restrictions within five carefully selected engineering, management and multidisciplinary databases, namely: Web of Science, Scopus, Inspec, Science direct and 
Compendex. The search was initially conducted in early 2018 and further refined in late 2019, using the following keywords (Phase 1 of Figure 2) in the subject topic, title and abstract fields: "industry* maintenance decision-making" OR "industry* maintenance decision making" OR "industry* maintenance multi-criteria decision support" OR "industry* maintenance multicriteria decision support" OR "industry* maintenance decision analysis" OR "industry* maintenance decision system" AND "manufacturing system*". These keywords significantly enhanced our ability to strike a good balance between search sensitivity and precision, without necessarily compromising the quality of the output information.

The initial search generated 950 articles from all databases (Phase 2 of Figure 2), which was subsequently refined to 323 after the application of relevant filters such as duplicates/triplicates/quadruplicates/quintuplicates removal, restriction of article types to journals and conference articles as well as including articles that are published in English language (Phase 3 of Figure 2). Further refinements of the outputs was then performed after classification according to discipline areas so as to determine those to be retained (Phase 4 of Figure 2). At this phase, it was determined that only 97 articles were relevant but only 76 of them were retained due to closely aligned or repeated contents. For instance, only 6 of the 42 articles within the engineering civil class were retained because the rest were more concerned with infrastructure maintenance decisions and not manufacturing. Finally, the performance of the search process was examined for sensitivity (i.e. percentage of relevant articles retrieved for the area of interest) and precision (i.e. percentage of retrieved articles that are aligned to the area of interest), which returned values of $78 \%$ and $24 \%$ respectively. The sensitivity and precision values are considered acceptable and near optimum threshold (Zhang et al., 2019; Zhou et al., 2015), which in turn satisfied the conditions of the search process.

According to the search process, some of the key research contributions include the development of a smart decision-making framework with the ability to select from several maintenance alternatives including minimal repair/replacement of damaged machine components or to perform preventive maintenance tasks (Cassady et al. 2001). The application of optimized decision making support for maintenance service operations was also proposed in the study conducted by Murthy et al. (1999), which was aimed at helping organisations discriminate between different maintenance strategies often proposed for different assets. Zavadskas et al. (2017) similarly applied a fuzzy logic based multiple criteria model to optimise facilities management strategies allocation. The model was adjudged to boost the confidence levels of facility management decision-makers, owing to the integration of three distinct utility functions (i.e. distance-to-ideal solution, additive utility function and multiplicative utility function). The possibilities of developing an approach that could potentially minimize mean-waitingtimes (MWT) or set-up times for periodic maintenance activities by deciding whether it is more costeffective to group tasks for various plant assets or individual allocation was investigated by Wijnmalen and Hontelez (1997), Wildeman et al. (1997) and van Dijkhuizen and van Harten (1997). 
Figure 2: Review process for trends in industrial maintenance decision-making

Further conscious efforts towards eliminating or at least minimizing the wastage of already scarce resources often associated with the generalization of maintenance strategies for all plant assets has also triggered ascent in the application of multiple criteria decision analyses. Multiple criteria decision analysis (MCDA) is a global term used for representing quantitative techniques that are capable of supporting multi-dimensional decision-making scenarios with multiple and/or conflicting objectives (Marttunen and Hämäläinen, 2008; Stirling, 2006; Geneletti, 2013; Han et al., 2019; Mahdi et al., 2019; Illankoon et al., 2019; Wang and Djurdjanovic, 2019; Fong et al., 2019). A typical example of a decision-making scenario for a remotely located cement manufacturing plant where MCDA could be very valuable is in deciding whether to stick with conventional PPM and maintain high kiln refractory bricks stock or implement CBM whereby bricks delivery is determined by wear rates. Obviously, each of these decisions possesses its impacts on finance, safety, downtime and environment regarded as desirable by some stakeholders and undesirable by others. For instance, the production manager is likely to support PPM and high stock levels because it offers a greater assurance to plant availability and hence higher throughput. The maintenance manager on the contrary might support CBM because it offers an opportunity to reduce the spares holding costs as well as maintenance workload variability. With regards to maintenance spare parts management, Cavalieri et al. (2008) developed a 5-staged (i.e. parts coding, parts classification, parts demand forecasting, stock management and validation) MCDA-based framework with a potential to systematically guide industrial managers on how to optimise their maintenance spares. Similarly, a more recent study by Driessen et al. (2015) focussed on the development of an MCDA-based planning and control framework for the maintenance spares of highvalue capital assets with the transport industry.

Further studies in MCDA for plant maintenance management include those conducted by Labib et al. (1996); Lazakis and Olcer (2016); Bevilacqua and Braglia (2000) and Van Horenbeek and Pintelon (2014). In these MCDA studies, the authors applied techniques such as analytic hierarchy and analytic network processes to facilitate the recommendation of cost-effective maintenance strategies based on the comparison of prior defined critical parameters such as downtime, failure frequency, spares cost, etc. Despite the usefulness of these earlier applications of MCDA, the analysis process that precedes the decision making often involves testing each of the considered maintenance strategies on each plant asset before eventual evaluation of suitability with MCDA. This approach at its best is time-consuming and could be unrealistic especially when dealing with industrial plants that are yet to possess a very rich database of performance variables. Based on these limitations, Ishizaka and Nemery (2014) explored the use of ELECTRE-SORT to allocate plant assets to difficult-to-compare maintenance strategies. The application of artificial intelligence (AI) based techniques such as fuzzy logic, artificial neural networks (ANN), support vector machines (SVM), etc., for MCDA of complex industrial production and 
maintenance activities has also been explored by several earlier researchers (Grant 1986, Randhawa and McDowell 1990, Kathawala and Allen 1993, Kanet and Adelsberger 1987, Byrd and Hauser Jr 1991). The primary rationale behind incorporating AI-based techniques or expert systems is the anticipated capability of such systems to adequately obtain human decision behaviours that have been previously perceived to be impalpable (Weirs 1997), so that organisations can minimise inconsistencies that originate from human errors. The study by Grant (1986) led to the development of a prototype scheduler that possessed the potential to enhance the efficiency of search strategies by modelling human expertise. More recent studies have also explored the use of AI based approaches to increase the general ease with which maintenance strategies are selected. Some examples include the use of fuzzy interactive linear assignment approach (Bashiri et al. 2011), fuzzy analytic network process (Pourjavad and Shirouyehzad 2014) and fuzzy multiple group approach (Lazakis and Ölçer 2016). In addition to the studies that specifically describe the characteristics of individual models, the current body of literature also contains ample and comprehensive surveys (Cho and Parlar 1991, Dekker 1996, Jardine and Tsang 2013, McCall 1965, Nakagawa 2006, Pharm and Wang 1996, Wang 2002) that are aimed at increasing the awareness of maintenance practitioners on the existence of different maintenance optimization models.

Hence, based on the current spate of information related to maintenance management decision optimization available, it is fair to assume that the challenge is not limited to concept development but rather how to effectively narrow the gap between theory and sustainable practice. The reasons for the fissure in knowledge transfer and standardization between academia and industry has been sparsely investigated by some researchers (Scarf 1997, Garg and Deshmukh 2006) who grouped these into three main categories. Firstly, a significant number of articles are solely developed for mathematical purposes which are often too complex to comprehend and/or implement by industry professionals that are mainly interested in finding logical but simplified solutions to persistent plant problems (Moya 2004, Wang 2002). Secondly, some academic researchers often focus too much attention on the development of new models for the sake of enriching their personal and institutional research profiles but far less on reallife applicability (Scarf 1997, Garg 2006). It can also be argued that in an attempt to justify the relevance of the new techniques proposed by some academic publications, far too much attention is focussed on exposing the limitations of earlier techniques and far less on their strengths which sometimes creates a shadow-chasing phenomenon and eventual loss of interest among industry experts. Thirdly, case studies in maintenance decision making and optimization are underrepresented in the current body of literature despite an overwhelming consensus that there should be a reasonable split between the theory and practice of maintenance (Nicolai and Dekker 2008, Van Horenbeek et al. 2010).

Moreover, when comparing theory to practice, there seems to be a bias in the literature towards oversimplifications in order to minimise complexity. This has been observed some years ago by reviewing the chasm between existing literature related to reliability and maintenance models versus 
what practitioners actually experience in the field of maintenance (Shorrocks and Labib 2000), and it was observed that there was a gap between them. The observed gaps were primarily due to three main factors: unawareness of decision makers about existing models, lack of demonstrable case studies related to impact, and the inherent unrealistic assumptions embedded in many reliability models. Some of these observations remain till today. For instance, most studies tend to focus on the efficiency of single equipment without considering the production configuration and reconfiguration issues (Abdi and Labib 2017). Some researchers (Alrabghi and Tiwari 2015, Chemweno et al. 2015) argue that systems compromising of a single machine producing a single product or two exactly identical machines are considered as a bias towards oversimplification and that such research does not really resemble the current complexity and interactions in real manufacturing systems. More so, assumptions such as maintenance resources (e.g. spare parts) being readily available and/or that every maintenance intervention always restores plant assets to as-good-as-new conditions have proven to be unrealistic in practice (Hu et al. 2017).

The need to address the highlighted concerns triggers the fundamental question of whether academic researchers should continue to be "slaves of complexity" by developing models that may never go beyond academic databases or "masters of simplicity" whereby proposed models have industrial applicability at their core. While the authors of this paper adequately acknowledge the enormity of the challenges especially the availability of reliable plant data, it is also important to recognise the fact that some infinitesimal traces of such standardized concepts already exist in certain aspects of manufacturing services. For instance, the application of frequency domain analysis, based on Fast Fourier transformation (FFT) for vibration based faults diagnosis (a vital aspect of CBM) is one of the most universally applied signal processing techniques across the industry and academia (Yunusakaltungo and Sinha 2014; Yunusa-Kaltungo et al., 2015; Luwei et al., 2018). Other notable examples include the potential-to-functional (PF) diagram (Andrawus et al., 2006; Andrawus et al., 2007) as well as the Pareto histograms. PF curves have been widely used to determine optimal maintenance intervention intervals for plant assets, based on parameters such as MTTR and downtime implication. Similarly, the Pareto histogram that was developed in the late $19^{\text {th }}$ century has been immensely used by researchers and industrial maintenance experts to ascertain maintenance priorities by ranking equipment failures according to their relative contributions to plant downtime (Knights 2004). Besides the versatility of these techniques, a fundamental reason for their universal and sustained acceptability over the decades is computational simplicity.

\section{Revisiting popular maintenance strategies}

While classification and fundamental understanding of maintenance strategies is not new to academic and industrial literatures, the lack of a truly standardized terminology for individual strategies still poses 
a challenge despite the attempts by regulatory bodies such as International Standards Organisation (ISO), British Standards Institute (BSI), Institute of Asset Management (IAM), European Standardisation Organisations (e.g. CEN, CENELEC or ETSI), etc. For instance, BM/reactive/run-tofailure/corrective maintenance; CBM; PPM/scheduled maintenance/preventive maintenance, etc. In fact, some authors have gone further to distinguish corrective maintenance from BM, arguing that the former initiates failure investigation mechanisms such as root cause or fault tree analysis (Yunusakaltungo et al. 2017; Cheung et al., 2019) immediately after the initial failure so as to prevent reoccurrence while the latter (i.e. BM) only focuses on repair/replace actions after individual failures. Irrespective of the choice of terminologies adopted, it must be ensured that such are consistent and embedded within the premise of continuous improvement. The aim of the authors in this section is not to pass a judgement on what terminologies are right or wrong, but to offer readers absolute clarity on the classifications adopted for the current study. Since this paper focuses on the propagation of a simplified approach to maintenance decision making through the harmonisation of known independently developed tools, additional maintenance strategies such as skills-level-upgrade (SLU) and design-out-maintenance (DOM) have also been included so as to concurrently address both efficiency and effectiveness. Hence the five maintenance strategies to be considered in this study are BM, PPM, CBM, DOM and SLU.

\section{$3.1 \quad B M$}

According to Seecharan et al. (2018) the breakdown (BM) strategy is applicable to machines with a track record of both low frequency of failures and low severity (downtime). This is the optimal state and is considered an optimised state where the performance of all existing asset should aim at. Therefore, BM is a suggested strategy that aims at sustaining best existing practice for the machines concerned. Note that BM does not imply ignoring the asset but rather the emphasis here is to audit sustainability of the existing best practice.

\section{$3.2 P P M$}

Planned preventive maintenance (PPM) is defined as preventative measures taken on a regular basis based on usage or calendar time. However, they differ in complexity since some of them represent "easy" PPM as proposed by Seecharan et al. (2018) and Moya (2004). For example, this may include who will execute the PPM tasks and when. Whereas what is considered as "difficult" PPM may then be restricted to the actual contents of the tasks. To be more illustrative, a simple PPM schedule for an asset such as a car, which may be as follows: "change the oil filter by the mechanic using a certain spanner every six months or every six thousand miles, whichever comes first". Now, this simple PPM instruction contains several features in terms of when or how often it takes place (every six months or every six thousand miles), who will carry it out (the mechanic as opposed to the driver), $\underline{\text { how }}$ it will be done (using a certain spanner), and $\underline{\boldsymbol{w h a t}}$ is the nature of the instruction itself (change the oil filter), Hence 
there are 'when?', 'who?' type of questions which are relatively easy to answer, and there are 'what?' and 'how?' type of questions which are relatively difficult to answer because they need more advanced expertise.

\section{$3.3 \quad C B M$}

Condition Based Maintenance (CBM) is a suitable strategy for assets that experience low frequency of failure but high downtime. Such assets can have catastrophic failures resulting in extended downtime ("show stoppers"). By monitoring their condition with such techniques as infrared thermography and vibration analysis, one should be able to pre-empt failures and proactively enact measures to avoid downtime. For a detailed review of CBM, see Jardine et al. (2006).

\section{$3.4 \quad$ Skills level upgrade (SLU)}

When breakdowns occur frequently but are fixed quickly resulting in little downtime, Labib et al. (1996) recommends that the SLU strategy should be used. One of the underpinning elements of total productive maintenance (TPM) is the transfer of some basic maintenance skills from maintenance technicians to the front-line operators in production, hence the term "productive maintenance" (Nakajima 1998, Hartmann 1992). Hence chronic losses (“death by a thousand cuts") should be dealt with using TPMtype initiatives (Seecharan et al. 2018). For example, menial tasks such as machine resetting or minor adjustments can be carried out by operators once they have received the correct training, i.e. SLU. Another variation of SLU is to de-skill the maintenance function on a machine by focusing on improving its maintainability.

\subsection{Design-out-maintenance (DOM)}

The DOM strategy is applicable to worst performing assets in both criteria of frequency and severity (downtime). Major design out projects need to be considered for any machines in this section. An asset in this quadrant is considered not fit for purpose, and hence it should be a candidate for next shut down, overhaul or turnaround activities.

\subsection{Summary statement on popular maintenance strategies}

In as much as maintenance literatures often advocate the suitability of certain strategies over others

which may be rightly so under certain circumstances, it is vital to note that a truly robust and costeffective maintenance management strategy in real-life scenarios would comprise of varying proportions of each strategy depending on the complexity and diversity of the plant assets. For example, an all-integrated cement process plant would typically adopt CBM for its rotary kilns' main drive gearboxes; PPM for limestone crusher blow bars hard-facing since they wear with the amount and size 
of limestone crushed; SLU for heavy fuel oil (HFO) pump bleeding/pressure regulation; BM for light bulbs that illuminate the pedestrian walkways and DOM when considering a change from high-energy consuming pneumatic transport systems to mechanical transport systems such as drag chains and bucket conveyors.

\section{Integration of existing DMG approaches for optimised decision analysis}

An integrated framework can occur at a strategic or an organisational level (Caputo et al. 2007; Tam and Price, 2008; Cheung et al., 2019), where each maintenance strategy can be the responsibility of a team in the maintenance department (function) as proposed by Seecharan et al. (2018). For example, one team can be responsible for sustaining and auditing best practice for machines (BM maintenance strategy), whereas another team may be responsible for preventive maintenance of machines (PPM strategy). Also, there can be separate teams for implementing CBM strategies, skills level upgrade (SLU) strategies and plant redesign projects (DOM strategy). In other words the suggested strategies can be used as an integrated framework for the allocation of machines to various maintenance teams and the performance monitoring of those machines by the respective teams within the organisational maintenance function. As has been discussed earlier, the main challenge facing maintenance decision makers isn't the identification or definition of the strategies themselves but how to simplify the optimization of their allocation to plant assets.

In the current study, we attempt to achieve a similar objective by integrating two relatively known tools namely Pareto histograms and decision making grid (DMG). A primary criterion for selecting these particular tools is their application simplicity, versatility and speed although it could be argued that these tools are not particularly new especially Pareto histograms that have existed for decades. While Pareto histograms are quite popular amongst industry professionals, the technique has also encountered its fair share of criticisms owing to its requirement to generate multiple histograms for comparing asset performance against different maintenance parameters (Knights 2004). For instance, a Pareto analysis based on downtime and failure frequencies would require one histogram for comparing the downtimes of different plant assets and another for frequencies which are often difficult to trend over time because the relative positions of failure codes may change between periods (knights 2004). On the contrary, DMG has the capability to compensate for the deficiencies of Pareto histograms by offering a single plot that adequately compares the considered maintenance parameters (e.g. downtime and frequency), which makes them suitable for better classification of maintenance priorities. Despite these potentials, very few publications are currently available in the literature whereby their practical applications are demonstrated through real-life case studies exists (Seecharan et al. 2018).

\subsection{The DMG as a revised Risk Matrix}


Although traditional risk matrices have been used by many organisations and consultants, they have also been criticised (Cox, 2008) as a concept. Traditional risk matrices are constructed as a combination of the two main dimensions of risk; severity versus probability of occurrence. In our case, severity is equivalent to downtime, whereas probability of occurrence is analogous to frequency. Such traditional risk matrices have been criticised (Cox, 2008) with respect to poor resolution in terms of weak consistency, betweenness, and consistent colouring, as well as suboptimal resource allocation. Briefly, the DMG addresses such concerns. By poor resolution, it implies a weakness in traditional risk matrices in terms of their subjectivity where they can assign similar ratings to quantitatively very different risks. The same author concludes by stating that a 'promising research direction may be to focus on placing the grid lines in a risk matrix to minimize the maximum loss from misclassified risks' Cox (2008). In the DMG, the boundaries are determined in an objective way and the main focus of this paper is to address this specific issue in more detail as we will see in the following sections. The DMG also offers suggestions of appropriate strategies that can lead to optimised resource allocations. Again, this will be demonstrated in the subsequent sections. In addition, the DMG maps the performance of critical machines and hence can help in monitoring the impact of the decisions taken in the form of improvement in the relative locations of the equipment in the grid as shown in the case study below.

\subsection{DMG boundaries determination}

A typical DMG is a means of classifying plant assets into one of nine sections, based on the combinations of downtimes and failure frequencies. Each DMG section represents one of the five maintenance strategies described in Section 3 as depicted by Figure 3. This study will not dwell much on the specifics of DMG, as such information have been extensively provided by Seecharan et al. (2018). However, details on how the different boundaries of the grid are determined will be provided. There are three basic approaches for determining the low/medium and medium/high frequency and downtime boundaries in a typical DMG. The first approach which assumes data homogeneity is based on calculating the difference $(\delta)$ between the highest $(h)$ and lowest $(l)$ values of either frequency or downtime (Fernandez et al. 2003). Let's assume that we denote the highest and lowest frequency values by $\left(x_{h}\right)$ and $\left(x_{l}\right)$ respectively, then:

$$
\delta=x_{h}-x_{l}
$$

The DMG1 boundaries can be estimated as:

$$
\begin{array}{r}
\text { low } / \text { medium }=\left(\frac{x_{h}-x_{l}}{3}\right)=\left(\frac{\delta}{3}\right) \\
\text { medium/high }=2\left(\frac{x_{h}-x_{l}}{3}\right)=\left(\frac{2 \delta}{3}\right)
\end{array}
$$


Since it is impossible to always guarantee data homogeneity in reality, a second method based on clustering was proposed by Tahir et al. (2009). In this approach, boundaries are fixed at the point of maximum difference between successive downtime and frequency values (after sorting the data in a descending order). For further illustration, let's assume that the constant interval, highest value and lowest value are respectively denoted by $C I, X_{h}$ and $X_{l}$, DMG2 range for the 3 criteria are:

$$
\begin{gathered}
C I=\left[\frac{X_{h}-X_{l}}{3}\right] \\
\text { High }_{\text {criterion }}=\left[\left(X_{h}\right),\left(X_{h}-\left[\frac{X_{h}-X_{l}}{3}\right]\right)\right] \\
\text { Medium }_{\text {criterion }}=\left[\left(X_{h}-\left[\frac{X_{h}-X_{l}}{3}\right]\right),\left(X_{h}-2\left[\frac{X_{h}-X_{l}}{3}\right]\right)\right] \\
\text { Low }_{\text {criterion }}=\left[\left(X_{h}-2\left[\frac{X_{h}-X_{l}}{3}\right]\right),\left(X_{l}\right)\right]
\end{gathered}
$$

The third approach involves the use of fuzzy logic to determine membership functions that are capable of defining low, medium and high regions for frequency and downtime, based on the perception of the maintenance decision maker (Seecharan et al. 2018). The first and second approaches to DMG boundaries estimation are most commonly applied owing to the degree of subjectivity sometimes associated with the definition of what the asset owner perceives as more important. Hence, the scope of this study lies solely within the premises of approaches 1 and 2 .

Figure 3: A typical DMG showing the boundaries and different maintenance strategies

\section{Background of the case study}

The incorporation of case studies into research provides an effective means of simplifying the visualization of sometimes complex real-life scenarios (Yunusa-kaltungo 2017, Leonard-Barton 1990, Lewis 1998). In this paper, the case study is a Northern Nigerian four-decade old all-integrated cement process plant with an annual capacity of approximately one million metric tonnes. By all-integrated we mean that every aspect of cement manufacturing process (i.e. mining of raw materials to the dispatch of finished products) is situated on the same site and owned by the same organisation. Hence it is fair to assume that maintenance management policy is common. The case study is divided into six main process stages as depicted by Figure 4. With the exception of the limestone crusher plant (LCP), each 
of the six process stages are made up of two similarly configured continuously-operating production lines. For example, BP1 and BP2 in Figure 4 respectively represent lines 1 and 2 within the burning plant. Despite the case study being configured to operate on a continuous basis, scenarios emerge whereby business plans are modified so that different process stages function independently. Such scenarios include:

Weather conditions: The sales cycle analysis regularly conducted by the marketing team indicates that rainfalls reduce cement sales in this region due to significantly lower construction activities. Therefore, the business model of the organisation recommends that fewer production activities are done downstream the burning plants (i.e. cement grinding and packing-dispatch plants) during rainy seasons. Hence the clinker (the main ingredient in cement) produced at the BP process stage can be sold to cement grinding plants in other regions of the country where rainfall is less, or exported to neighbouring countries such as Niger, Chad and Cameroun or even stockpiled in the plant to supplement grinding during extended BP shutdowns.

Plant shutdowns: The annual maintenance plan recommends two 14-days shutdowns for each of process lines within the BP process stage (i.e. BP1 and BP2). During these shutdowns, fresh clinker production is halted but grinding of stockpiled clinker continues at the cement mills. Also, the lignite produced from the solid fuel grinding plants (SFGP1 AND SFGP2) can be sold to power stations.

Figure 4: Case study process flowchart

Overview of the underlying problem: The availability of a typical cement manufacturing company is usually dictated by the availability of its most critical process stages. According to the company's maintenance objectives for the three years considered, the availability targets for the most critical plants were set at 70\% (LCP), 94\% (SFGP) and 96\% (BP) but these targets were hardly met. The BP process stage outperforms all other stages despite an average loss of $2 \%$ availability. The performances of LCP and SFGP process stages were consistently poor over the periods ( $20 \%$ and $12 \%$ loss in availability respectively). The correlation between frequent plant stoppages and other plant performance factors (e.g. maintenance workload, cost, throughput, safety, etc.) cannot be over emphasized. As an illustration, stoppages at SFGP when cement demand is high would result to one of two scenarios. At best, clinker production at BP will continue on the basis of substitute low pour fuel oil (LPFO) which can increase energy cost by as much as $66 \%$. Under the worst case scenario, unavailability of LPFO would result to total loss of clinker production which could attract a daily downtime cost of approximately $£ 450,000$. Figure 5 shows a record of the key maintenance performance indicators for the critical process stages (LCP, BP and SFGP) over three years (2014-2016). In addition to the revenue losses that plagued such performances, plant interviews revealed that maintenance personnel are often 
in the fire-fighting mode as a result of the significant downtime hours across the critical plants. Hence there was little or no time for developing a framework that systematically segregates maintenance strategies according to individual asset performance.

Figure 5: Maintenance performance indicators for critical plants between 2014 and 2016

\subsection{Case study based harmonisation of DMG approaches}

While it is well known that the cornerstone of DMG is to emphasize the application of appropriate maintenance strategies that would counter modes of failures that impede overall effectiveness, the effects of the variations that exist between the methods leading to the estimation of its most important elements (i.e. low/medium and medium/high boundaries) have never been investigated by any of the previous studies. Rather, the primary focus of past DMG studies (Seecharan et al. 2018, Fernandez et al. 2003, Tahir et al. 2009) have mostly been centred on creating a dichotomy that is often based on the perceived supremacy of one approach over the other. The current study however adopts a contrary view that attempts to explore the possibility of integrating the collective strengths of both so as to increase the robustness and flexibility of strategies to maintenance decision-makers. In order to practically demonstrate simplicity and applicability, the initial stage involved the extraction of the three worst performing process stages (LCP, SFGP and BP) of the all-integrated manufacturing process shown in Figure 4 from the computerised maintenance management system (CMMS). The second phase of the analysis was based on the application of the principles of Pareto analysis to rank and select the most impacting failures as illustrated by Tables 1-3. It should be noted that the decision to limit this study to only 20 failure codes is purely based on convenience. The third phase of the analysis involved the mapping of the different failure codes into the appropriate DMG sections (Figure 6), based on the most popular DMG approaches which have been denoted as DMG1 and DMG2 for the purpose of simplicity.

Table 1: CMMS extracts of top 20 LCP failures

Table 2: CMMS extracts of top 20 SFGP failures

Table 3: CMMS extracts of top $20 \mathrm{BP}$ failures 
Figure 6: Comparison of maintenance strategy allocation for critical manufacturing process stages using DMG. (a) DMG1- LC (b) DMG2 - LC (c) DMG1 - SFGP (d) DMG2 - SFGP (e) DMG1 - BP (f) DMG2 - BP

\subsection{Existing plant-based strategies versus DMG}

According to Figure 6, the proposed maintenance strategies for the failure codes associated with BP (Figure 6(e)-(f)) were in total agreement. In order to adequately visualise the usefulness of combining both approaches in practice, reference was made to the plant's inspection job plan (IJP) and Table 4 provides a comparison between existing and proposed maintenance strategies as well as their implications to the cost-effectiveness of the operations. Using the worst performing plant (i.e. LCP) for illustration, it can be seen that BM strategy was recommended for failure codes LC4-LC7, LC14-LC16, LC18 and LC20 by DMGs 1 and 2. On the contrary, the current maintenance strategy in the plant's IJP is dominated by CBM and PPM activities for the same failure codes which would significantly increase maintenance costs (e.g. instrumentation and cost of manpower for experienced CBM analyst).

Table 4: Comparisons between DMG and existing LCP maintenance strategies

In addition, some of the failure codes are not worthy of CBM activities owing to their historically short repair times. As a practical illustration, failure codes LC5, LC6, LC7 and LC15 respectively relate to belt clip joints, tail drum rotational speed change sensor, sampler roller and chute. Based on a combination of a decade long maintenance experience of one of the authors of the current paper as well as probing in-house maintenance plans, it was quite clear that the decision to allocate CBM strategy to plant assets was mainly driven by the availability of a measurement parameter (e.g. vibration, speed, temperature, etc.) and far less on the implication of the failure of such assets on downtime and/or frequency. The IJP indicated that typical CBM inspection (i.e. a combination of first level and specific) visits to the LCP accounts for approximately 20 man-hours a week (excluding the time required for data analysis and report entry). With the current emphasis on the incorporation of strategic maintenance management principles by a significant number of organisations, the restriction of high-expertise-andexperience oriented maintenance activities such as CBM and DOM to the neediest assets is fast becoming a critical factor for cost-effectiveness. Besides the potentially huge and unjustifiable costs associated with indiscriminate implementation of CBM for most of the plant assets, manpower planning data also revealed that the maintenance workload and skills distribution was significantly skewed towards monitoring and less towards execution. Hence, the maintenance execution teams are unable to implement some of the required actions which in turn lead to the unplanned failures of critical plant assets such as the apron feeder. Table 4 also shows that the plant's maintenance strategy for LC1 (limestone crusher chamber) and LC3 (apron feeder) failure codes differs from those recommended by 
the DMGs. The currently implemented plant's maintenance strategy is BM, despite the colossal impacts of these failure codes on overall plant downtime. On the contrary, both DMGs recommend DOM for LC1. For LC3, DMG2 similarly recommends DOM as opposed to the PPM suggested by DMG1.

Both DMG approaches as discussed in Equations 1-4 in Section 4.1 are not mutually exclusive. In other words each one has its own applicability depending on the main objective and nature of data. For example, if the main objective is to use the DMG to organise the Maintenance Department within a certain organisation then as suggested by Seecharan et al. (2018), each maintenance strategy can be the responsibility of a team in the maintenance department (function). So in this case it can be argued that DMG1 is a better approach for classification as the main objective, in this case, will be to make every team 'busy' in working on an equipment that meets their expertise (function). For example, one team can be responsible for sustaining and auditing best practice for machines in the BM region, with another team responsible for preventive maintenance of machines in the PPM region. There can be teams for both reliability-centred maintenance (RCM) and CBM investigative approaches, a team for total productive maintenance (TPM) and upgrading maintenance skills strategies, and a team for major overhauls and redesign projects of machines in the DOM quadrant. In short, the DMG model can be used as a framework for the allocation of machines to various maintenance teams and the performance monitoring of those machines by the respective teams. Hence using DMG1 approach will make sure that there is at least more than one machine in most of the quadrants of the grid. On the other hand, if there is a large variation in the data about some machines compared to others in terms of the criteria of frequency or downtime, then DMG2 method will be more applicable to use in this case, as we make machines more comparable. So for example, if the worst failing machine tends to be experiencing a significant amount of downtime and frequency compared to all other machines in the list, then it is more reasonable to decide that only that machine would be in the High-High region of the DMG (i.e. DOM), which means a candidate for next shut down, as compared to all other machines. We have provided Table 4 which compares the strategies recommended by each DMG method to the real-life strategy in the case study plant based on the first author's experience of the plant as well as information that we obtained from the inspection job plan and verbal interviews.

\subsection{The potential role of DMG in futuristic maintenance management planning}

There is a current drive for the maintenance function to actively contribute to smart industries through concepts such as autonomous maintenance and internet of things (IoT). The cornerstone of these emerging concepts is based on reliable historical data which is readily retrievable from most in-house CMMS (Carnero and Noves 2006, Levrat et al. 2008, Takata et al. 2004). Most CMMS are designed to enhance the ability of maintenance experts in monitoring critical transactions and work flows (Levrat et al. 2008), but do they directly support maintenance decision-making? It is universally accepted that information is often considered an asset, but the non-conversion of copious quantities of information 
into decisions in real time can potentially choke the decision-making process. It is well-known that most CMMSs are built on a framework that allows integration of maintenance with other critical manufacturing functions including stores, production, finance, workshops, purchasing, etc. (Bradshaw, 2004; Kunche et al., 2012; Yunusa-Kaltungo and Sinha, 2017). Hence, the incorporation of a decisionmaking module such as DMG could become a step in the direction of filling the so-called "CMMS decision-making black hole." While it might still be early days, continued and extensive investigation of the potential of the technique through more industry-based studies could be very useful. Figure 7 attempts to illustrate how a simplified DMG-based MCDA sub menu can be incorporated into the existing maintenance interface of typical CMMS and e-Maintenance platforms.

Figure 7: Conceptual framework of the potential role of DMG within IoT.

The process commences with an automatic transfer of reliability data (mainly downtime and failure frequencies) from the maintenance main menu onto the DMG-based MCDA sub menu at a predetermined time T. The algorithm then ranks the data sets using the well-known Pareto principle. Based on the ranked downtime and failure frequency data, the DMG boundaries will be computed by the algorithm which then forms the basis for classification of individual failure codes into the sections corresponding to the appropriate maintenance strategies. In order to foster continuous improvement, information on the allocated strategies is then feedback into the CMMS so as to update and optimise the activities of other interface menus. For instance, such information could aid the warehouse identify what spares level to retain for different plant assets; provide information to new works/projects on the assets that would require redesigning in the future (short, mid and long terms); provide information on manpower planning; etc. If the organisation comprises of several sites such as in the chosen case study, information and knowledge from one site (e.g. cement manufacturing plant A) can be deposited onto a central maintenance strategy planning database which can be accessed by all other sites.

\section{Concluding remarks}

There have been earlier publications on the concepts of DMG but the main novelty of this paper is in applying the DMG approach in a different context, where we were able to demonstrate the applicability of its different methods of classification in the different process stages of a cement manufacturing company. In this paper, we have attempted to implement a relatively simple approach and demonstrated its applicability to a real industrial case study. Such 'simplicity' is evidenced by the following attributes; limited types and amount of data required and its availability, the limited number of assumptions involved, and integration of different available maintenance strategies. In terms of the amount of data required, which are frequency of breakdown events and the downtime for each event, such information 
is readily available in all CMMS that are currently implemented in most organisations, especially that we rely on just having access to some sort of a counter of faults and a timer for each event. There are very limited assumptions involved in the process as both DMG1 and DMG2 ways of classification depend on the main objective and nature of data involved. Additionally, we demonstrated the ability to integrate various maintenance strategies into a cohesive and systematic framework. Finally, the current study also provided a conceptual framework that stipulates how DMG could be integrated into maintenance management systems as well as the currently trending internet of things (IoT). It may still be adjudged early days, owing to unresolved issues still limiting full deployment of IoT such as big data management, data silo issues, data security and the protection of intellectual property of all stakeholders. However, the conceptual framework described here could still offer a blueprint and new lines of thinking especially owing to the fact that most of its integrated techniques are simple as well as contain elements (e.g. Pareto analysis) that already exist to some degree within the industry.

While the current study adequately demonstrates the practical implementation of the proposed hybrid DMG approach on an all-integrated cement manufacturing process, it can also be argued that all of the considered assets are limited to the same industry within the same geographical location. Hence, the perception and acceptance of risks within the chosen case study may be viewed as monotonic. Therefore, future lines of thoughts for improving the confidence levels of this approach will be centred on expanding the study scope to a wider collection of production processes (e.g. oil and gas, power, transport, etc.) with different risk priority levels and data distribution. It is adequately acknowledged that the difference in failure data types across different industries would significantly increase data dimensionality, which could further increase big data management challenges of IoT. Therefore, we intend to explore the incorporation of dimensionality reduction tools such as principal (PCA) or independent components analyses (ICA) for input data rationalisation, without necessarily compromising the quality of the output.

\section{References}

Abdi, M. R., \& Labib, A. (2017). RMS capacity utilisation: product family and supply chain. International Journal of Production Research, 55(7), 1930-1956.

Aguezzoul, A. (2014). Third-party logistics selection problem: A literature review on criteria and methods. Omega, 49, 69-78.

Al-Najjar, B. (2007). The lack of maintenance and not maintenance which costs: A model to describe and quantify the impact of vibration-based maintenance on company's business. International Journal of Production Economics, 107(1), 260-273.

Alrabghi, A., \& Tiwari, A. (2015). State of the art in simulation-based optimisation for maintenance systems. Computers \& Industrial Engineering, 82, 167-182. 
Andrawus, J. A., Watson, J., Kishk, M., \& Adam, A. (2006). The selection of a suitable maintenance strategy for wind turbines. Wind Engineering, 30(6), 471-486.

Andrawus, J. A., Watson, J., \& Kishk, M. (2007). Wind turbine maintenance optimisation: principles of quantitative maintenance optimisation. Wind Engineering, 31(2), 101-110.

Aslam-Zainudeen, N. \& Labib, A. (2011). Practical application of the decision making grid (DMG). Journal of Quality in Maintenance Engineering, 17(2), pp.138-149.

Ayu Nariswari NP, Bamford D, Dehe B. Testing an AHP model for aircraft spare parts. Production Planning \& Control. 2019 Mar 12;30(4):329-44.

Bashiri, M., Badri, H., \& Hejazi, T. H. (2011). Selecting optimum maintenance strategy by fuzzy interactive linear assignment method. Applied Mathematical Modelling, 35(1), 152-164.

Bevilacqua, M., \& Braglia, M. (2000). The analytic hierarchy process applied to maintenance strategy selection. Reliability Engineering \& System Safety, 70(1), 71-83.

Botti, L., \& Peypoch, N. (2013). Multi-criteria ELECTRE method and destination competitiveness. Tourism Management Perspectives, 6, 108-113.

Bradshaw, I. (2004). Bradshaw, L., 2004. Improved CMMS and asset management systems. Maintenance Journal, 17(2), pp.7-14.

BS 3811. (1984). Glossary of maintenance terms in terotechnology.

Bumblauskas, D., Gemmill, D., Igou, A., \& Anzengruber, J. (2017). Smart Maintenance Decision Support Systems (SMDSS) based on corporate big data analytics. Expert Systems with Applications, 90, 303-317.

Byrd, T. A., \& Hauser Jr, R. D. (1991). Expert systems in production and operations management: possible human side effects in implementation. Production and Inventory Management Journal, 32(4), 53.

Cai H, Liu Y, Wu H, Ma P, Hu A. Equipment Maintenance Mode Decision Based on Fuzzy Multiattribute Decision Method. In Recent Trends in Intelligent Computing, Communication and Devices 2020 (pp. 123-129). Springer, Singapore.

Caputo, A. C., \& Pelagagge*, P. M. (2005). Introduction of an integrated maintenance system in the tissue paper sector: a case study. Production Planning \& Control, 16(3), 243-254.

Carnero, M. C., \& Novés, J. L. (2006). Selection of computerised maintenance management system by means of multicriteria methods. Production Planning and Control, 17(4), 335-354.

Cassady, C. R., Murdock Jr, W. P., \& Pohl, E. A. (2001). Selective maintenance for support equipment involving multiple maintenance actions. European Journal of Operational Research, 129(2), 252-258.

Cavalieri, S., Garetti, M. A. R. C. O., Macchi, M. A. R. C. O., \& Pinto, R. O. B. E. R. T. O. (2008). A decision-making framework for managing maintenance spare parts. Production planning \& control, 19(4), 379-396.

Chan, F. T. S., Lau, H. C. W., Ip, R. W. L., Chan, H. K., \& Kong, S. (2005). Implementation of total productive maintenance: A case study. International Journal of Production Economics, 95(1), 71-94. 
Chemweno, P. K., Pintelon, L., \& Muchiri, P. N. (2015). Evaluating the impact of spare parts pooling strategy on the maintenance of unreliable repairable systems. IFAC-PapersOnLine, 48(3), 989-994.

Cheung CM, Yunusa-Kaltungo A, Ejohwomu O, Zhang RP. 15 Learning from failures (LFF). Construction Health and Safety in Developing Countries. 2019 Sep 12.

Chilcott, J. B., \& Christer, A. H. (1991). Modelling of condition based maintenance at the coal face. International Journal of Production Economics, 22(1), 1-11.

Cho, D. I., \& Parlar, M. (1991). A survey of maintenance models for multi-unit systems. European Journal of Operational Research, 51(1), 1-23.

Corrente, S., Greco, S., \& Słowiński, R. (2013). Multiple criteria hierarchy process with ELECTRE and PROMETHEE. Omega, 41(5), 820-846.

Cowton, C. J., \& Vail, R. L. (1994). Making sense of just-in-time production: a resource-based perspective. Omega, 22(5), 427-441.

Cox LA Jr 2008. What's wrong with risk matrices? Risk Analysis 28, no. 2 (2008): 497-512.

Dekker, R. (1996). Applications of maintenance optimization models: a review and analysis. Reliability engineering \& system safety, 51(3), 229-240.

Dong M, Zheng H, Zhang Y, Shi K, Yao S, Kou X, Ding G, Guo L. A novel maintenance decision making model of power transformers based on reliability and economy assessment. IEEE Access. 2019 Feb 5;7:28778-90.

Driessen, M., Arts, J., van Houtum, G.J., Rustenburg, J.W. \& Huisman, B. (2015). Maintenance spare parts planning and control: a framework for control and agenda for future research. Production Planning \& Control, 26(5), pp.407-426.

Terminology, M. (2001). European Standard. CEN (European Committee for Standardization), EN, 13306.

Fernandez, O., Labib, A. W., Walmsley, R., \& Petty, D. J. (2003). A decision support maintenance management system: development and implementation. International Journal of Quality \& Reliability Management, 20(8), 965-979.

Fong JT, Filliben JJ, Heckert NA, Leber DD, Berkman PA, Chapman RE. Uncertainty Quantification of Failure Probability and a Dynamic Risk Analysis of Decision-Making for Maintenance of Aging Infrastructure. In Risk Based Technologies 2019 (pp. 65-80). Springer, Singapore.

Garg, A., \& Deshmukh, S. G. (2006). Maintenance management: literature review and directions. Journal of quality in maintenance engineering, 12(3), 205-238.

Geneletti, D. (2013). Assessing the impact of alternative land-use zoning policies on future ecosystem services. Environmental Impact Assessment Review, 40, 25-35.

Goh YM, Micheler S, Sanchez-Salas A, Case K, Bumblauskas D, Monfared R. A variability taxonomy to support automation decision-making for manufacturing processes. Production Planning \& Control. 2019 Jul 30:1-7. 
Grant, T. J. (1986). Lessons for OR from AI: A scheduling case study. Journal of the Operational Research Society, 37(1), 41-57.

Guillén, A.J., Crespo, A., Macchi M., \& Gómez, J. (2016). On the role of Prognostics and Health Management in advanced maintenance systems. Production Planning \& Control, Volume 27, 2016 Issue 12.

Han C, Fang M, Ma T, Cao H, Peng H. An intelligent decision-making framework for asphalt pavement maintenance using the clustering-PageRank algorithm. Engineering Optimization. 2019 Nov 6:1-9.

Herrera Galán M. Evidence-based asset management applied to maintenance function control. Journal of Quality in Maintenance Engineering. 2019 Apr 17.

Hartmann, E. (1992). Successfully installing TPM in a non-Japanese plant: total productive maintenance. TPM Press.

Hatami-Marbini, A., \& Tavana, M. (2011). An extension of the Electre I method for group decisionmaking under a fuzzy environment. Omega, 39(4), 373-386.

Hines, P., Holweg, M., \& Rich, N. (2004). Learning to evolve: a review of contemporary lean thinking. International journal of operations \& production management, 24(10), 994-1011.

Holweg, M. (2007). The genealogy of lean production. Journal of operations management, 25(2), 420 437.

Hu, Q., Chakhar, S., Siraj, S., \& Labib, A. (2017). Spare parts classification in industrial manufacturing using the dominance-based rough set approach. European Journal of Operational Research, 262(3), 1136-1163.

Illankoon P, Tretten P, Singh S. Decision Support System for Flight Maintenance Technicians: Issues and Challenges. eMaintenance. 2019:88.

Ishizaka, A., \& Nemery, P. (2014). Assigning machines to incomparable maintenance strategies with ELECTRE-SORT. Omega, 47, 45-59.

Jafari, L., \& Makis, V. (2015). Joint optimal lot sizing and preventive maintenance policy for a production facility subject to condition monitoring. International Journal of Production Economics, 169, 156-168.

Jardine, A. K., Lin, D., \& Banjevic, D. (2006). A review on machinery diagnostics and prognostics implementing condition-based maintenance. Mechanical systems and signal processing, 20(7), 14831510.

Jardine, A. K., \& Tsang, A. H. (2013). Maintenance, replacement, and reliability: theory and applications. CRC press.

Kanet, J. J., \& Adelsberger, H. H. (1987). Expert systems in production scheduling. European Journal of Operational Research, 29(1), 51-59.

Kathawala, Y., \& Allen, W. R. (1993). Expert systems and job shop scheduling. International Journal of Operations \& Production Management, 13(2), 23-35. 
Knights, P. F. (2004). Downtime priorities, Jack-knife diagrams, and the business cycle. Maintenance Journal, 17(2), 14-21.

Krafcik, J. F. (1988). Triumph of the lean production system. MIT Sloan Management Review, 30(1), 41.

Kubiak, W., \& Yavuz, M. (2008). Just-in-time smoothing through batching. Manufacturing \& Service Operations Management, 10(3), 506-518.

Kunche, S., Chen, C., \& Pecht, M. (2012). A review of PHM system's architectural frameworks. In The 54th Meeting of the Society for Machinery Failure Prevention Technology, Dayton, $\mathrm{OH}$.

Labib, A., Williams, G., \& O'Connor, R. (1996). Formulation of an appropriate productive maintenance strategy using multiple criteria decision making. Maintenance-Farnham, 11(2), 14-21.

Lazakis, I., \& Ölçer, A. (2016). Selection of the best maintenance approach in the maritime industry under fuzzy multiple attributive group decision-making environment. Proceedings of the Institution of Mechanical Engineers, Part M: Journal of Engineering for the Maritime Environment, 230(2), 297309.

Leonard-Barton, D. (1990). A dual methodology for case studies: Synergistic use of a longitudinal single site with replicated multiple sites. Organization science, 1(3), 248-266.

Levrat, E., Iung, B., \& Crespo Marquez, A. (2008). E-maintenance: review and conceptual framework. Production Planning \& Control, 19(4), 408-429.

Lewis, M. W. (1998). Iterative triangulation: a theory development process using existing case studies. Journal of Operations Management, 16(4), 455-469.

Li H, Shang J, Shen Z, Zhang D. Research on Network Level Pavement Maintenance Decision-Making Based on Matter-Element Model. In CICTP 2019 Jul (pp. 763-775).

Lin L, Wang F, Luo B. An optimization algorithm inspired by propagation of yeast for fleet maintenance decision making problem involving fatigue structures. Applied Soft Computing. 2019 Dec $1 ; 85: 105755$.

Luwei KC, Yunusa-Kaltungo A, Sha'aban YA. Integrated Fault Detection Framework for Classifying Rotating Machine Faults Using Frequency Domain Data Fusion and Artificial Neural Networks. Machines. 2018 Nov 20;6(4):59.

Mahdi IM, Khalil AH, Mahdi HA, Mansour DM. Decision support system for optimal bridge'maintenance. International Journal of Construction Management. 2019 Jun 7:1-5.

Marquez, A. C., \& Gupta, J. N. (2006). Contemporary maintenance management: process, framework and supporting pillars. Omega, 34(3), 313-326.

Marttunen, M., \& Hämäläinen, R. P. (2008). The decision analysis interview approach in the collaborative management of a large regulated water course. Environmental Management, 42(6), 1026.

McCall, J. J. (1965). Maintenance policies for stochastically failing equipment: a survey. Management science, 11(5), 493-524. 
Melton, T. (2005). The benefits of lean manufacturing: what lean thinking has to offer the process industries. Chemical engineering research and design, 83(6), 662-673.

Moya, M. C. C. (2004). The control of the setting up of a predictive maintenance programme using a system of indicators. Omega, 32(1), 57-75.

Murthy, D. N. P., \& Asgharizadeh, E. (1999). Optimal decision making in a maintenance service operation. European Journal of Operational Research, 116(2), 259-273.

Nachimuthu S, Zuo MJ, Ding Y. A Decision-making Model for Corrective Maintenance of Offshore Wind Turbines Considering Uncertainties. Energies. 2019 Jan;12(8):1408.

Nakagawa, T. (2006). Maintenance theory of reliability. Springer Science \& Business Media.

Nakajima, S. (1988). Introduction to TPM: Total Productive Maintenance.(Translation). Productivity Press, Inc., 1988, 129.

Nicolai, R. P., \& Dekker, R. (2008). Optimal maintenance of multi-component systems: a review. In Complex system maintenance handbook (pp. 263-286). Springer, London.

Papadopoulos, A., \& Karagiannidis, A. (2008). Application of the multi-criteria analysis method Electre III for the optimisation of decentralised energy systems. Omega, 36(5), 766-776.

Pourjavad, E., \& Shirouyehzad, H. (2014). Evaluating manufacturing systems by fuzzy ANP: a case study. International Journal of Applied Management Science, 6(1), 65-83.

Randhawa, S. U., \& McDowell, E. D. (1990). An investigation of the applicability of expert systems to job shop scheduling. International Journal of Man-Machine Studies, 32(2), 203-213.

Roda I, Macchi M, Albanese S. Building a Total Cost of Ownership model to support manufacturing asset lifecycle management. Production Planning \& Control. 2019 Jun 6:1-9.

Savsar, M. (2006). Effects of maintenance policies on the productivity of flexible manufacturing cells. Omega, 34(3), 274-282.

Scarf, P. A. (1997). On the application of mathematical models in maintenance. European Journal of operational research, 99(3), 493-506.

Seecharan, T., Labib, A., \& Jardine, A. (2018). Maintenance strategies: Decision Making Grid vs JackKnife Diagram. Journal of Quality in Maintenance Engineering, 24(1), 61-78.

Shahin, A., Aminsabouri, N. and Kianfar, K., 2018. Developing a Decision Making Grid for determining proactive maintenance tactics: A case study in the steel industry. Journal of Manufacturing Technology Management, 29(8), pp.1296-1315.

Shahin A, Labib A, Emami S, Karbasian M. Improving Decision-Making Grid based on interdependence among failures with a case study in the steel industry. The TQM Journal. 2019 Mar 4;31(2):167-82.

Shorrocks, P., \& Labib, A. W. (2000, November). Towards a multimedia based decision support system for word class maintenance. In Proceedings of the 14th Advances in Reliability Technology Symposium, University of Manchester. 
Silver, E. A. (2004). Process management instead of operations management. Manufacturing \& Service Operations Management, 6(4), 273-279.

Stirling, A. (2006). Analysis, participation and power: justification and closure in participatory multicriteria analysis. Land use policy, 23(1), 95-107.

Tahir, Z., Burhanuddin, M. A., Ahmad, A. R., Halawani, S. M., \& Arif, F. (2009, December). Improvement of decision making grid model for maintenance management in small and medium industries. In Industrial and Information Systems (ICIIS), 2009 International Conference on (pp. 598603). IEEE.

Takata, S., Kirnura, F., van Houten, F. J., Westkamper, E., Shpitalni, M., Ceglarek, D., \& Lee, J. (2004). Maintenance: changing role in life cycle management. CIRP Annals-Manufacturing Technology, 53(2), 643-655.

Tam, A. S., \& Price, J. W. (2008). A generic asset management framework for optimising maintenance investment decision. Production Planning \& Control, 19(4), 287-300.

van Dijkhuizen, G., \& van Harten, A. (1997). Optimal clustering of frequency-constrained maintenance jobs with shared set-ups. European Journal of Operational Research, 99(3), 552-564.

Van Horenbeek, A., Pintelon, L., \& Muchiri, P. (2010). Maintenance optimization models and criteria. International Journal of System Assurance Engineering and Management, 1(3), 189-200.

Van Horenbeek, A., \& Pintelon, L. (2014). Development of a maintenance performance measurement framework-using the analytic network process (ANP) for maintenance performance indicator selection. Omega, 42(1), 33-46.

Vujanović, D., Momčilović, V., Bojović, N., \& Papić, V. (2012). Evaluation of vehicle fleet maintenance management indicators by application of DEMATEL and ANP. Expert Systems with Applications, 39(12), 10552-10563.

Wang K, Djurdjanovic D. Joint Optimization of Preventive Maintenance and Spare Parts Logistics for Multi-echelon Geographically Dispersed Systems. In Asset Intelligence through Integration and Interoperability and Contemporary Vibration Engineering Technologies 2019 (pp. 643-653). Springer, Cham.

Wang, H. (2002). A survey of maintenance policies of deteriorating systems. European journal of operational research, 139(3), 469-489.

Wang, L., Chu, J., \& Wu, J. (2007). Selection of optimum maintenance strategies based on a fuzzy analytic hierarchy process. International journal of production economics, 107(1), 151-163.

Wang, W., Hussin, B., \& Jefferis, T. (2012). A case study of condition based maintenance modelling based upon the oil analysis data of marine diesel engines using stochastic filtering. International Journal of Production Economics, 136(1), 84-92.

Wang, X., \& Triantaphyllou, E. (2008). Ranking irregularities when evaluating alternatives by using some ELECTRE methods. Omega, 36(1), 45-63.

Wiers, V. C. (1997). A review of the applicability of OR and AI scheduling techniques in practice. Omega, 25(2), 145-153. 
Wijnmalen, D. J., \& Hontelez, J. A. (1997). Coordinated condition-based repair strategies for components of a multi-component maintenance system with discounts. European Journal of Operational Research, 98(1), 52-63.

Wildeman, R. E., Dekker, R., \& Smit, A. C. J. M. (1997). A dynamic policy for grouping maintenance activities. European Journal of Operational Research, 99(3), 530-551.

Wireman, T. (1990). World class maintenance management(No. 713). Industrial Pr.

Womack, J. P., Jones, D. T., \& Roos, D. (1990). The Machine that Changed the World: The Story of Lean Production HarperCollins Publishers. New York, USA.

Yunusa-Kaltungo, A., Kermani, M. M., \& Labib, A. (2017). Investigation of critical failures using root cause analysis methods: Case study of ASH cement PLC. Engineering Failure Analysis, 73, 25-45.

Yunusa-Kaltungo, A., \& Sinha, J. K. (2014). Combined bispectrum and trispectrum for faults diagnosis in rotating machines. Proceedings of the Institution of Mechanical Engineers, Part O: Journal of Risk and Reliability, 228(4), 419-428.

Yunusa-Kaltungo A, Sinha JK. Faults diagnosis in rotating machines using higher order spectra. In ASME turbo expo 2014: turbine technical conference and exposition 2014 Jun 16. American Society of Mechanical Engineers Digital Collection.

Yunusa-Kaltungo A, Sinha JK. Sensitivity analysis of higher order coherent spectra in machine faults diagnosis. Structural Health Monitoring. 2016 Sep;15(5):555-67.

Yunusa-Kaltungo A, Sinha JK, Nembhard AD. Use of composite higher order spectra for faults diagnosis of rotating machines with different foundation flexibilities. Measurement. 2015 Jun 1;70:4761.

Yunusa-Kaltungo A, Sinha JK. Effective vibration-based condition monitoring (eVCM) of rotating machines. Journal of Quality in Maintenance Engineering. 2017 Aug 14;23(3):279-96.

Yunusa-Kaltungo A, Sinha J. Condition monitoring: a simple and practical approach. LAP Lambert Academic Publishing; 2012.

Yurin, A. Y. (2012). An approach for definition of recommendations for prevention of repeated failures with the aid of case-based reasoning and group decision-making methods. Expert Systems with Applications, 39(10), 9282-9287.

Zavadskas, E. K., Turskis, Z., Vilutienè, T., \& Lepkova, N. (2017). Integrated group fuzzy multi-criteria model: Case of facilities management strategy selection. Expert Systems with Applications, 82, 317331.

Zhang H, Huang X, Zhou X, Huang H, Babar MA. Ethnographic research in software engineering: a critical review and checklist. In Proceedings of the 2019 27th ACM Joint Meeting on European Software Engineering Conference and Symposium on the Foundations of Software Engineering 2019 Aug 12 (pp. 659-670). ACM.

Zhou Y, Zhang H, Huang X, Yang S, Babar MA, Tang H. Quality assessment of systematic reviews in software engineering: A tertiary study. In Proceedings of the 19th international conference on evaluation and assessment in software engineering 2015 Apr 27 (p. 14). ACM. 
Zhu J, Bian L, Ding T. Study on Method and Decision Making Model for Preventive Maintenance Planning of Asphalt Pavement. In CICTP 2019 Jul (pp. 740-750). 


\section{List of Tables}

Table 1: CMMS extracts of top 20 LCP failures

\begin{tabular}{|c|c|c|c|c|}
\hline Plant Equipment & Failure Code & Failure Description & Downtime & Frequency \\
\hline Limestone crusher chamber & LC1 & Boulder jammed on jack & 131.74 & 93 \\
\hline \multirow{3}{*}{ Apron feeder } & LC2 & Apron feeder plate crack & 60 & 38 \\
\hline & LC3 & Apron feeder spillage & 70 & 59 \\
\hline & LC19 & speed regulator & 41 & 1 \\
\hline \multirow{2}{*}{ Limestone crusher rotor } & LC4 & Primary rotor trip & 7.31 & 12 \\
\hline & LC18 & Secondary rotor trip & 2.68 & 7 \\
\hline \multirow{4}{*}{ Belt A } & LC5 & Failed belt clips at joint & 1.25 & 1 \\
\hline & LC9 & Overload - OCR & 27.61 & 9 \\
\hline & LC20 & Reclaim belt side skirting & 0.5 & 1 \\
\hline & LC10 & Belt A rocon & 3.5 & 21 \\
\hline Belt B & LC12 & Misalignment & 16.97 & 1 \\
\hline Belt C & LC11 & Tail drum failure & 3.67 & 1 \\
\hline \multirow{8}{*}{ Stacker } & LC6 & Belt $\mathrm{C}$ tail drum rocon & 0.3 & 1 \\
\hline & LC7 & Sampler roller failure & 0.67 & 1 \\
\hline & LC8 & Traverse gear failure & 15.87 & 3 \\
\hline & $\mathrm{LC} 17$ & Boom belt overload & 11.79 & 13 \\
\hline & LC16 & Boom belt brake failure & 4.3 & 1 \\
\hline & $\mathrm{LC} 13$ & Hanger bearing failure & 31.23 & 1 \\
\hline & $\mathrm{LC} 14$ & Trip wire bracket failure & 0.3 & 1 \\
\hline & LC15 & Cracks on chute & 4.42 & 3 \\
\hline
\end{tabular}

Table 2: CMMS extracts of top 20 SFGP failures

\begin{tabular}{ccccc}
\hline Plant Equipment & Failure Code & Failure Description & Downtime & Frequency \\
\hline \multirow{2}{*}{ FK pump } & SF8 & Damaged gland & 17.05 & 1 \\
\cline { 2 - 5 } Bag house & SF20 & Barrel failure & 40.54 & 7 \\
& SF10 & Hopper level sensor & 1.15 & 17 \\
\cline { 2 - 5 } Ball mill & SF1 & CO analyser & 1.7 & 2 \\
& SF2 & Liner plates failure & 32 & 1 \\
\cline { 2 - 5 } Mill main drive & SF11 & Blocked diaphragm slots & 16.85 & 2 \\
\cline { 2 - 5 } & SF3 & Low lube oil pump pressure & 3.47 & 1 \\
\cline { 2 - 5 } & SF16 & High gearbox temperature & 0.85 & 2 \\
\hline Drag chain conveyor & SF6 & ISSB temperature sensor & 5.23 & 4 \\
\hline Bag filter & SF4 & Drag chain jammed & 10.64 & 6 \\
\hline Compressor & SF5 & High differential pressure & 2.37 & 3 \\
\hline Instrument air & SF7 & Low discharge pressure & 0.45 & 1 \\
compressor & SF9 & Process cooling water & 66.85 & 17 \\
\hline Fan & SF12 & Trip & 13.49 & 2 \\
\hline Weigh feeder & SF13 & Belt misalignment & 2.72 & 3 \\
\hline Screw conveyor & SF14 & Trip & 4 & 5 \\
\hline Diverter & SF15 & Damper diversion & 39.42 & 1 \\
\hline Coal surge bin & SF17 & Pre-bin level sensor failure & 0.85 & 11 \\
\hline Raw coal hopper & SF18 & Faulty raw coal indication & 0.43 & 1 \\
\hline Blower & SF19 & High vibration & 2.08 & 2 \\
\hline
\end{tabular}


Table 3: CMMS extracts of top 20 BP failures

\begin{tabular}{|c|c|c|c|c|}
\hline Plant Equipment & Failure Code & Failure Description & Downtime & Frequency \\
\hline & BP6 & GA compressor air pressure & 0.5 & 1 \\
\hline & & Station 2 roller bearing & & \\
\hline & BP2 & temperature & 31.42 & 1 \\
\hline & BP19 & Discharge chute blockage & 5.42 & 1 \\
\hline Coolers & BP7 & Cooler saddle pin & 0.75 & 1 \\
\hline \multirow{2}{*}{ Firing system } & BP16 & Kiln oil flow loss & 0.33 & 1 \\
\hline & BP18 & Firing fan impeller & 0.25 & 1 \\
\hline \multirow{2}{*}{ Protection } & BP1 & Low speed indication & 0.33 & 1 \\
\hline & BP17 & Uphill limit switch & 0.5 & 1 \\
\hline Bag filter & BP3 & By-pass damper jammed close & 0.5 & 1 \\
\hline \multirow{2}{*}{ Final fan } & BP9 & Final fan tripped on overload & 0.25 & 1 \\
\hline & BP15 & Final fan motor & 2.09 & 2 \\
\hline Extraction air slide & BP11 & Atomizer drag chain & 11 & 1 \\
\hline \multirow{2}{*}{ Clinker buckets } & BP10 & Clinker bucket chute blockage & 0.17 & 1 \\
\hline & BP13 & Clinker bucket derailment & 0.13 & 1 \\
\hline Gepol fan & BP20 & Trip & 0.33 & 1 \\
\hline \multirow{2}{*}{ Shell protection } & BP4 & High kiln shell temperature & 2.17 & 1 \\
\hline & BP8 & Refractory failure & 69.5 & 1 \\
\hline Generator & BP12 & Speed governor & 33.32 & 10 \\
\hline \multirow{3}{*}{ Feed transport } & BP14 & LIW RMT FK pump overload & 0.88 & 18 \\
\hline & & LIW power cylinder jammed & & \\
\hline & BP5 & close & 0.5 & 1 \\
\hline
\end{tabular}

Table 4: Comparisons between DMG and existing LCP maintenance strategies

\begin{tabular}{|c|c|c|c|}
\hline \multirow[b]{2}{*}{ Failure code } & \multirow{2}{*}{$\begin{array}{c}\text { Current Maintenance } \\
\text { Strategy }\end{array}$} & \multicolumn{2}{|c|}{ DMG Strategy } \\
\hline & & DMG1 & DMG2 \\
\hline LC1 & $\mathrm{BM}$ & \multicolumn{2}{|c|}{ DOM } \\
\hline LC2 & $\mathrm{BM}$ & \multicolumn{2}{|c|}{ PPM } \\
\hline LC3 & $\mathrm{BM}$ & PPM & DOM \\
\hline LC4 & CBM & \multirow{4}{*}{\multicolumn{2}{|c|}{$\mathrm{BM}$}} \\
\hline LC5 & PPM & & \\
\hline LC6 & $\mathrm{CBM}$ & & \\
\hline LC7 & CBM & & \\
\hline LC8 & CBM & BM & PPM \\
\hline LC9 & $\mathrm{BM}$ & $\mathrm{BM}$ & PPM \\
\hline LC10 & CBM & $\mathrm{BM}$ & PPM \\
\hline LC11 & CBM & \multicolumn{2}{|c|}{ BM } \\
\hline LC12 & $\mathrm{BM}$ & BM & PPM \\
\hline $\mathrm{LC} 13$ & CBM & $\mathrm{BM}$ & PPM \\
\hline LC14 & CBM & \multirow{3}{*}{\multicolumn{2}{|c|}{$\mathrm{BM}$}} \\
\hline LC15 & CBM & & \\
\hline LC16 & CBM & & \\
\hline LC17 & $\mathrm{BM}$ & BM & PPM \\
\hline LC18 & CBM & \multicolumn{2}{|c|}{$\mathrm{BM}$} \\
\hline LC19 & PPM & $\mathrm{BM}$ & PPM \\
\hline LC20 & & & \\
\hline
\end{tabular}




\section{List of Figures}

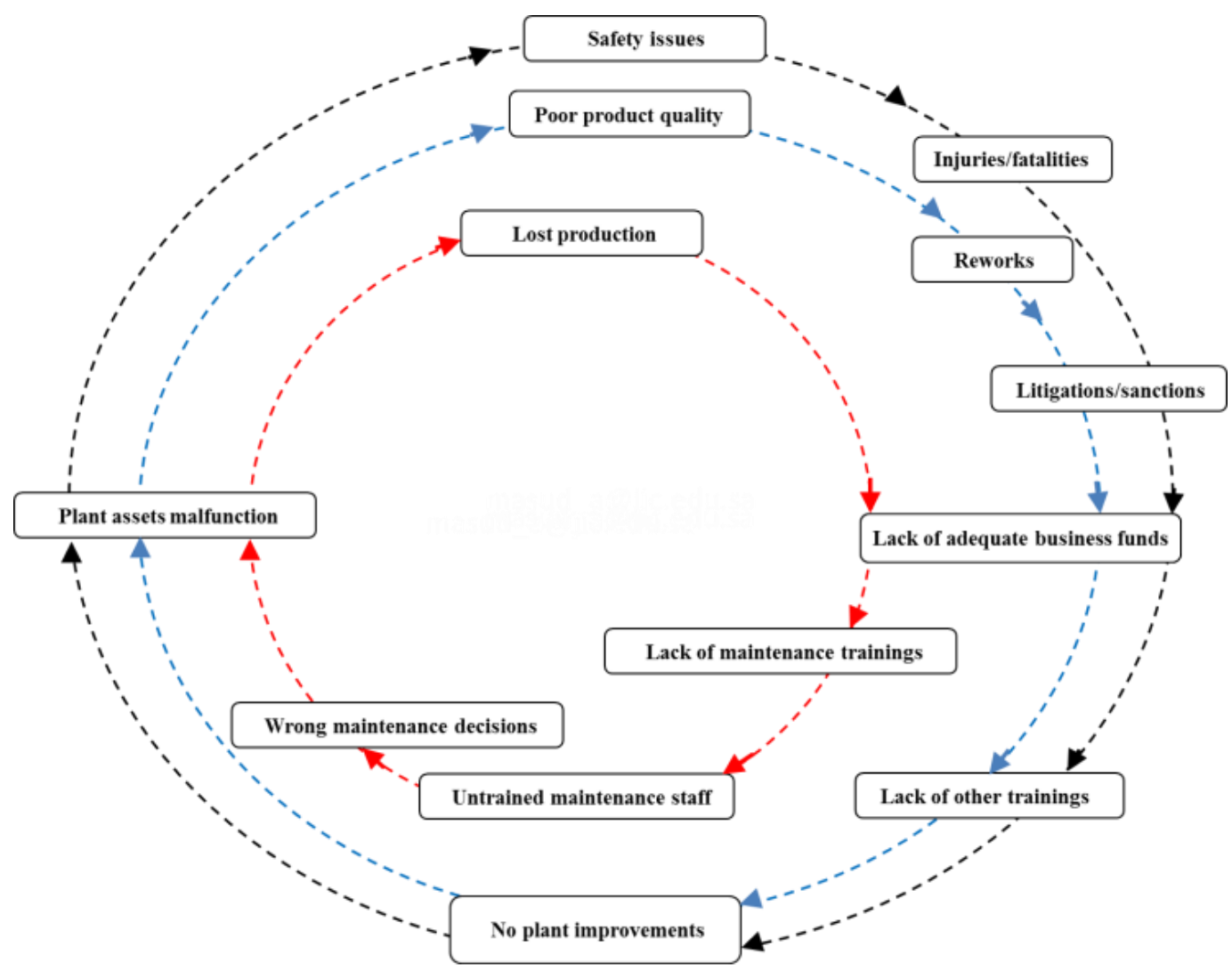

Figure 1: A generic maintenance management causal loop 


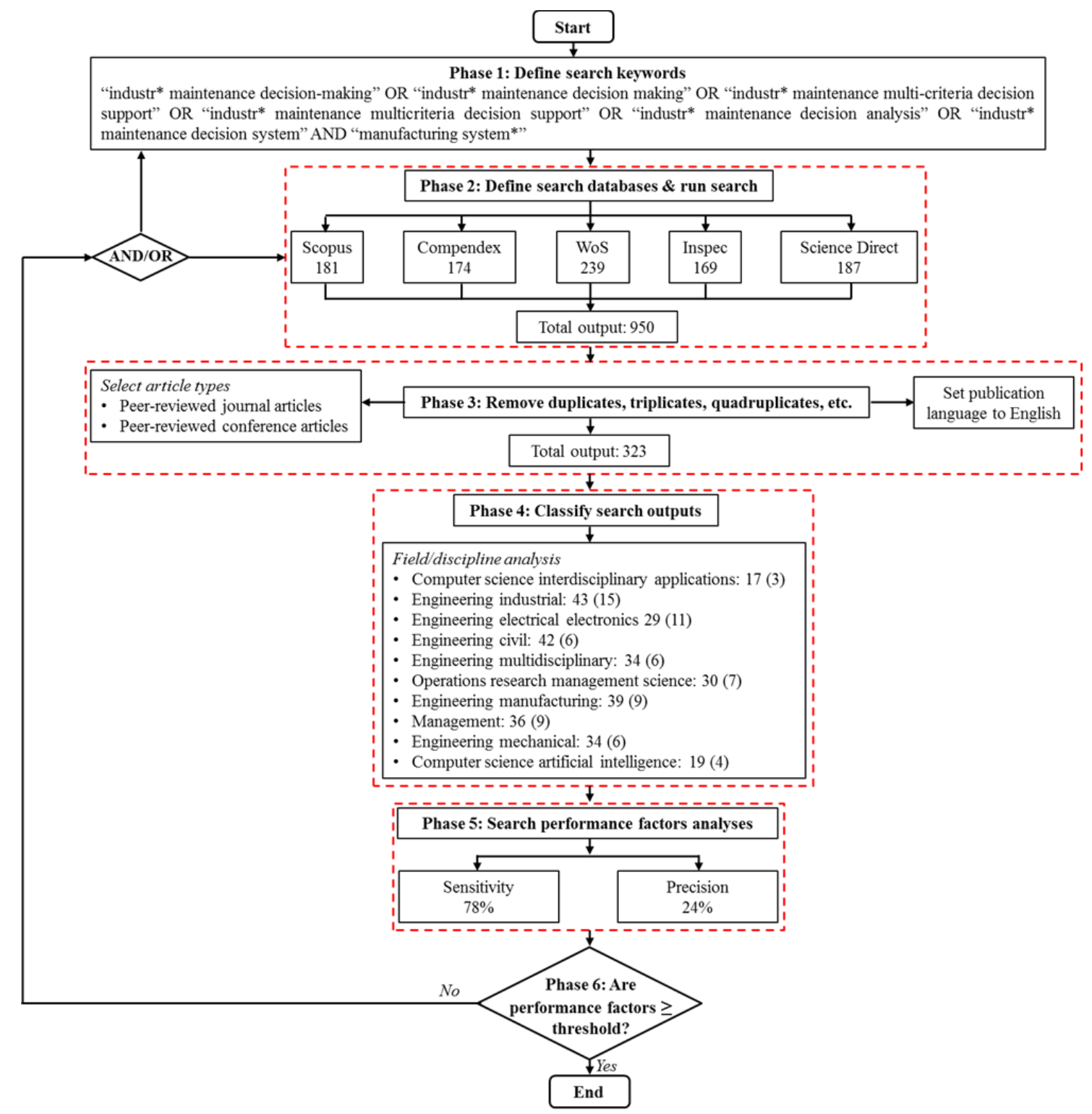

Figure 2: Review process for trends in industrial maintenance decision-making 


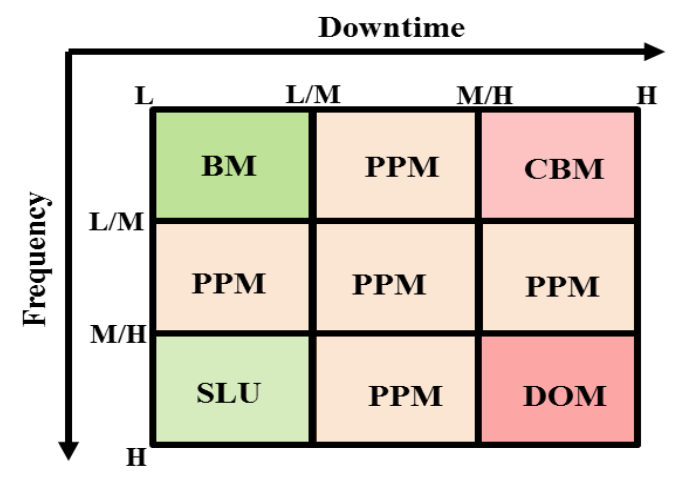

Figure 3: A typical DMG showing the boundaries and different maintenance strategies

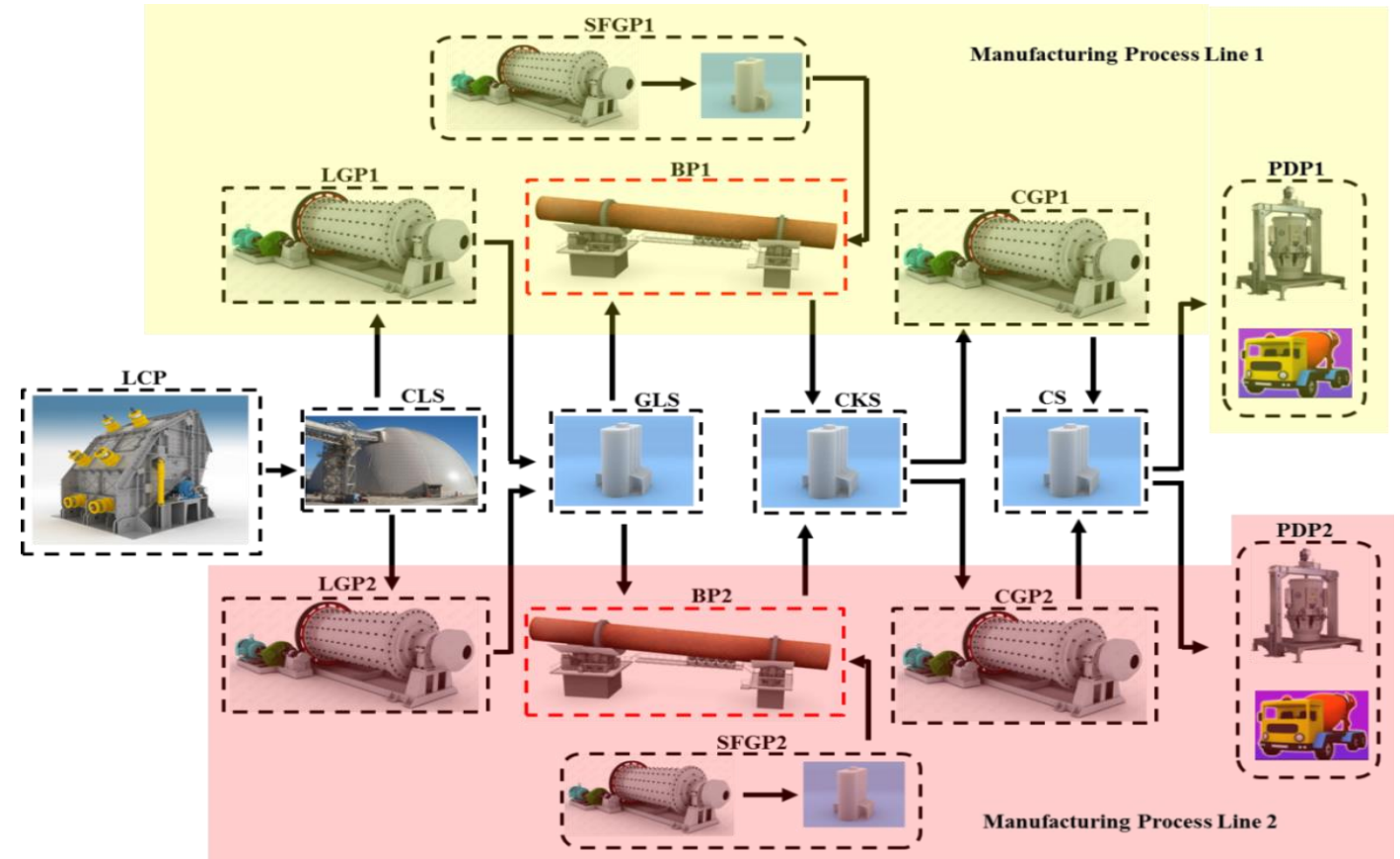

* Limestone crusher plant (LCP), crushed limestone storage (CLS), ground limestone storage (GLS), clinker storage (CKS), cement storage (CS), limestone grinding plants (LGP1 \& LGP2), burning plants (BP1 \& BP2), solid fuel grinding plants (SFGP1 \& SFGP2), cement grinding plants (CGP1 \& CGP2) and packing-dispatch plants (PDP1 \& PDP2).

Figure 4: Case study process flowchart 


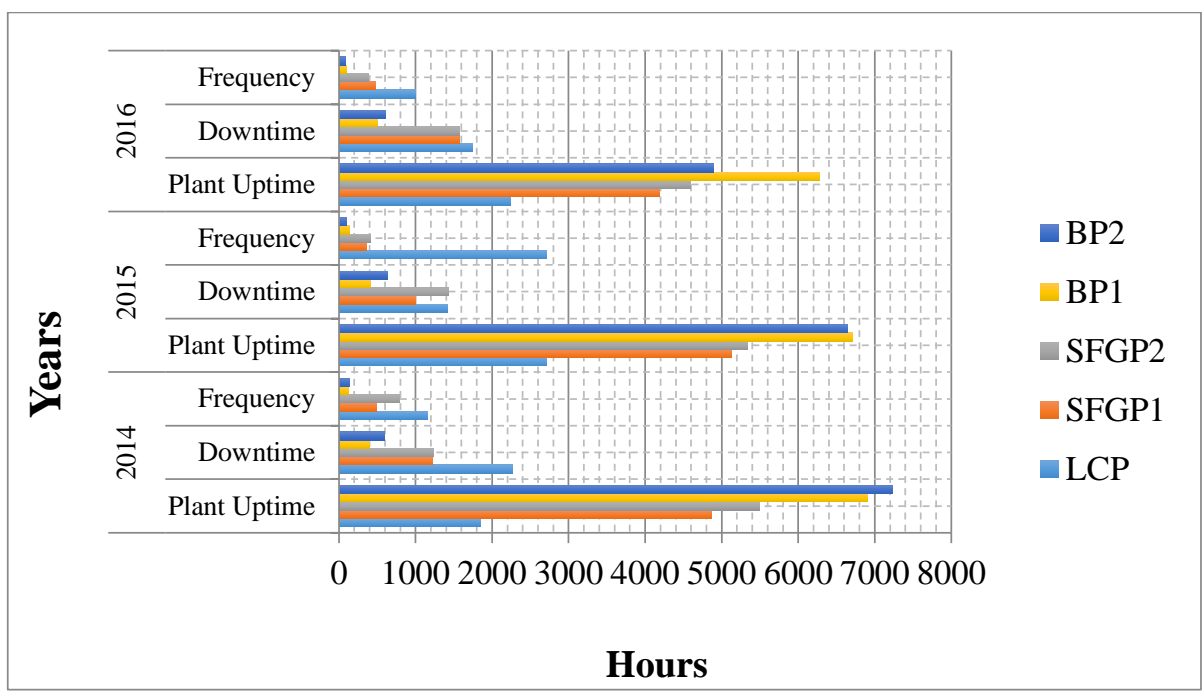

Figure 5: Maintenance performance indicators for critical plants between 2014 and 2016 

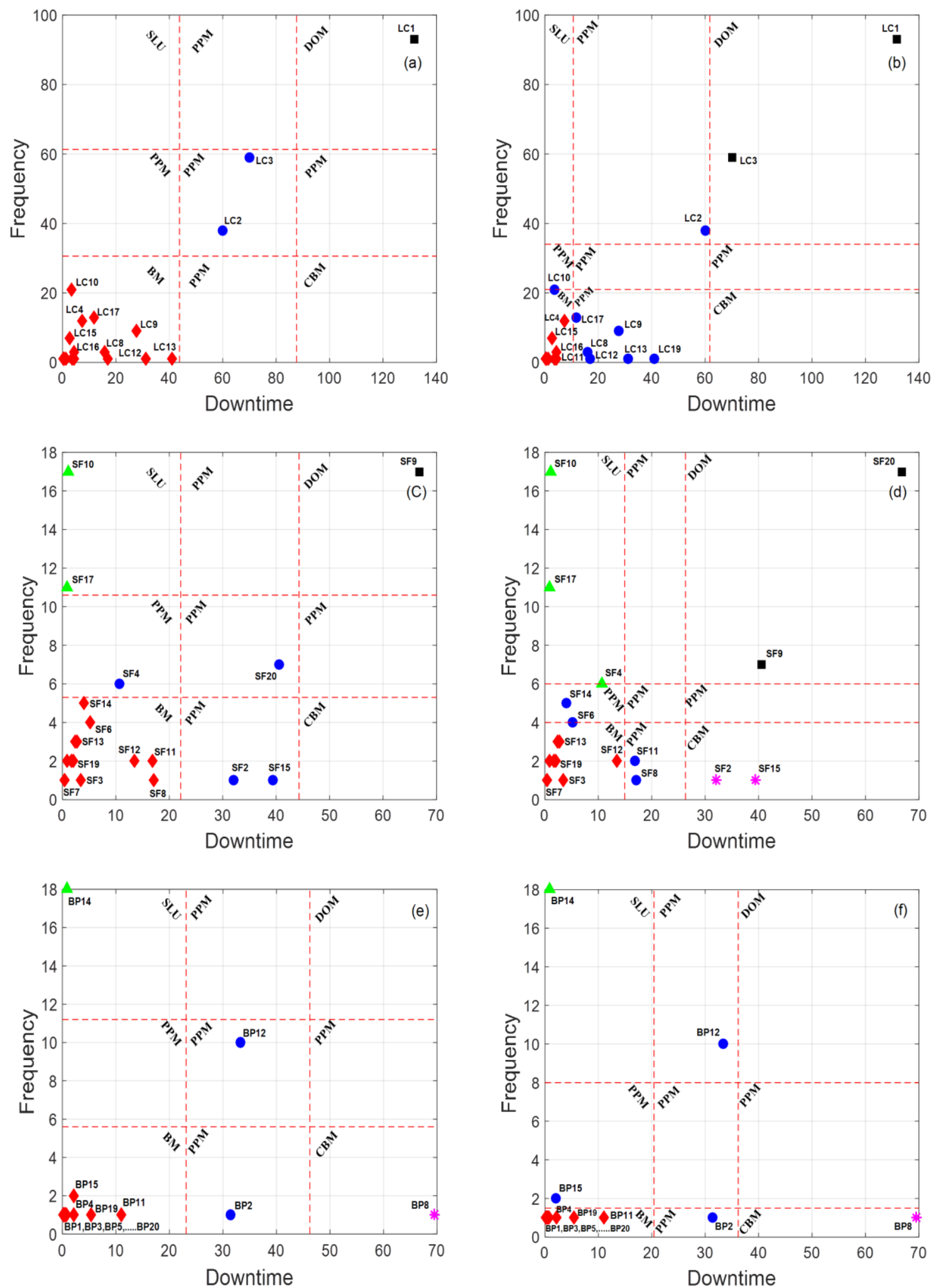

- BM - PPM $\triangle$ SLU - DOM * CBM

Figure 6: Comparison of maintenance strategy allocation for critical manufacturing process stages using DMG. (a) DMG1- LC (b) DMG2 - LC (c) DMG1 - SFGP (d) DMG2 - SFGP (e) DMG1 - BP (f) DMG2 - BP 


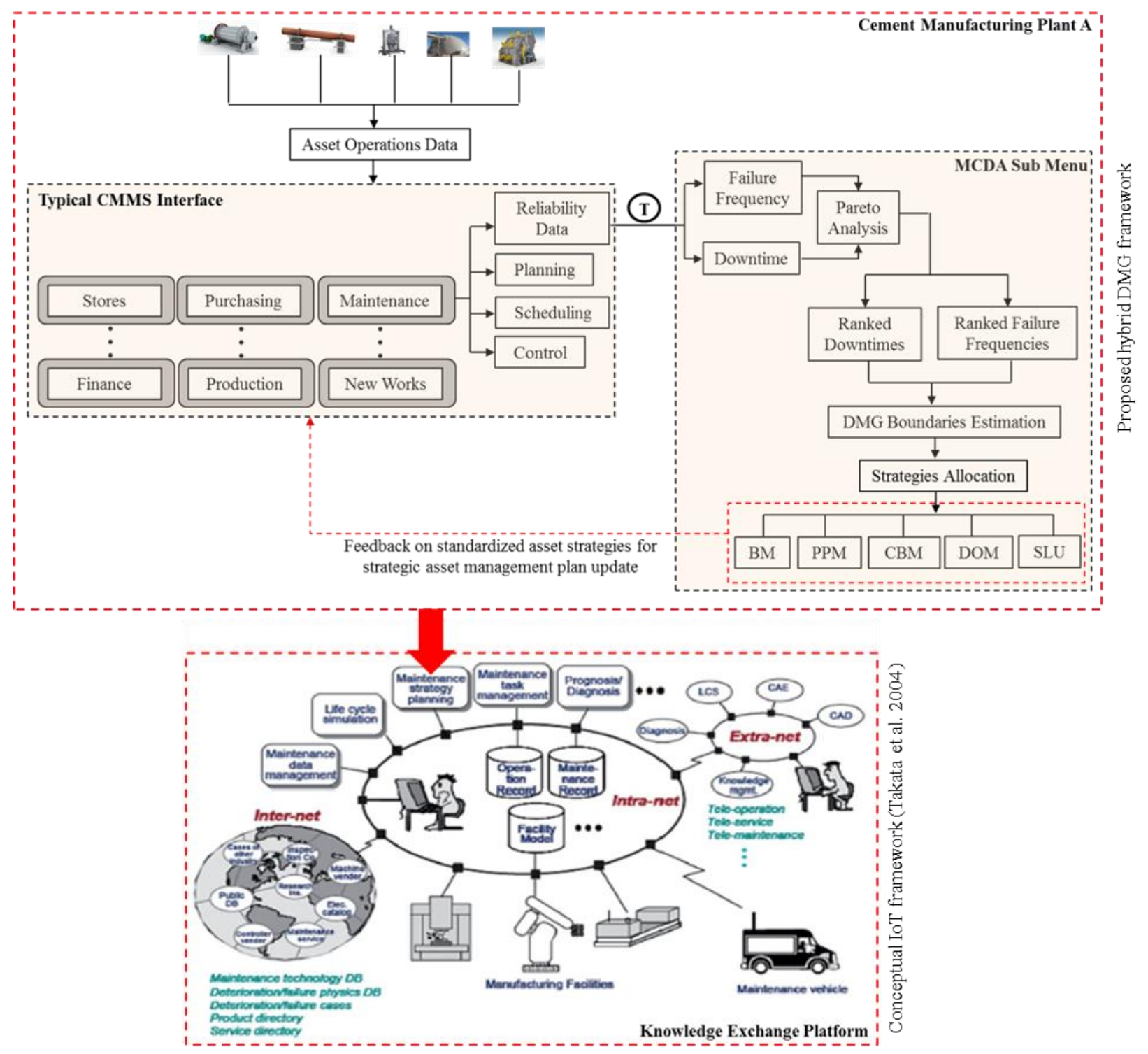

Figure 7: Conceptual framework of the potential role of DMG within IoT. 